\title{
A norm-aware multi-agent system for social simulations in a river basin
}

\author{
Ignasi Gómez-Sebastià ${ }^{1}$, Luis Oliva-Felipe ${ }^{1}$, Ulises Cortés ${ }^{1}$, Marta Verdaguer $^{2}$, \\ Manel Poch ${ }^{2}$ and Ignasi Rodríguez-Roda ${ }^{3}$ \\ ${ }^{1}$ Knowledge Engineering and Machine Learning Group. Universitat Politècnica de Catalunya \\ (UPC) C/Jordi Girona 1-3, E-08034 , Barcelona, Spain. \\ igomez@cs.upc.edu,loliva@cs.upc.edu,ia@cs.upc.edu \\ ${ }^{2}$ Laboratori d'Enginyeria Química i Ambiental (LEQUIA), University of Girona, 17071 \\ Girona, Spain manel@lequia.udg.cat \\ ${ }^{3}$ Laboratori d'Enginyeria Química i Ambiental (LEQUIA), University of Girona, 17071 \\ Girona, Spain ignasi@lequia.udg.cat Institut Català de Recerca de l'Aigua (ICRA), Emili \\ Grahit 101, 17003 Girona, Spain irodriguezroda@icra.cat
}

\begin{abstract}
Wastewater management is a complex task involving a wide range of technical enviromental and social factors. Furthermore, it typically requires the coordination of a heterogeneous society of actors with different goals. Regulations and protocols can be effectively used to tackle this complexity. In this chapter we present a norm-aware multi-agent system for social simulations in a river basin. The norms we present are inspired in European policies for wastewater management and they can evolve through time.
\end{abstract}

\section{Introduction}

In this chapter we will study the effect of regulations in the administration of wastewater fluxes in a river basin. Wastewater management is a difficult task, requiring the simultaneous consideration of a wide range of factors: technical, environmental, economical, social, legal, etc. When applied to a scenario such as a river basin, wastewater management requires to coordinate a wide range of activities performed by a society of actors with different goals (sometimes not aligned with the holistic goal of the society). The combination of these factors results in the fact that wastewater management in river basin is a complex process. One of such scenarios, for instance, involve the well-known dilemma of the Tragedy of the Commons (Hardin 1968), where rational agents use fresh water as part of their operations thus generating wastewater. Left alone, these agents would overpass wastewater treatment plants capacity until river gets too polluted and, hence, no longer usable by anybody.

Regulations and protocols are one of the possible solutions we can apply to tame complexity. However, due to the implicit complexity of the scenario it is hard for 
policy makers to tailor the exact set of regulations that will govern the system. On the one hand, several interacting factors have to be taken into account when designing the regulations. On the other hand, such regulations cannot be tested in real scenarios due to the cost of their application and its environmental implications.

We present a norm-aware multi-agent system (MAS) for social simulations in a river basin. Our system allows studying the effect of regulations in the behaviour of the different actors involved in a scenario based on the Tragedy of the Commons, as well as analysing different agents' behaviours under such scenario. The idea is using a norm-aware simulator where policies encourage the alignment of agents' behaviour to the common objective of the system, effectively detecting and sanctioning free-raiders and misbehaving actors, thus trying to prevent the Tragedy. The policies we present are inspired in European policies for wastewater management practice. Furthermore, policies in our system can evolve through time as a result of agents' behaviour and adapt to unexpected situations such as heavy rains or river pollution. Information technologies applied to environmental issues show potential in a wide range of fields, among others, decision support systems (Poch et al. 2004) and simulations (Hamilton 1969). However, the complexity of environmental problems introduces several challenges that information technologies should tackle. The first one is the fact that environmental issues must be considered in terms of complex systems, mainly due to the amount of variables to be considered and their dependencies. Also the high degree of uncertainty associated to the system and the potential impact (and therefore, risk) of the decisions taken w.r.t. these systems. The second issue is the fact that, in environmental systems, the scenario should often reflect conflicting goals, and we need to take into account a set of heterogeneous (sometimes conflicting) views and perspectives. These complex scenarios, where a wide variety of actors with different (sometimes conflicting) goals interact between them, can benefit from norm-aware electronic distributed systems based on agent technologies. Such systems can ensure compliance with the different actors to the expected behaviours and environmental policies, where environmental policies are designed to guide the overall system to a common higher goal, such as the preservation of the environment while keeping an active economy.

Our proposal is a norm-aware agent-based model for integrated Wastewater Management Systems. We apply this to the Besòs River Basin. The idea of using Autonomous Agents to cope with the problem has been done in view of the various, sometimes conflicting, goals that the identified actors have to fulfil their private interests. In this scenario each actor requires its own system view with customized privileges and access to differing control tools, either managerial or operational.

The system proposed aims at managing the treatment capabilities of Wastewater Treatment Plants (WWTPs), allowing coordination among them and with the different actors in the scenario. Plants treat wastewater coming from various sources before discharging it to the river, treated and with the appropriate ecologic conditions. To ensure river's ecological quality, water sensors measuring different parameters (e.g., temperature, acidity, suspended solids on water, river oxygen demand, etc.) are located along the river in interesting points, such as water 
discharge points for WWTPs. Plants are connected to various elements providing wastewater with different characteristics, including: towns providing household wastewater with a steady flow of quantity and variable pollutant concentrations; rain retaining tanks providing lightly polluted meteo wastewater, which comes in very high quantities during short periods of time; industries providing industrial wastewater with high variability both in quantity and pollutant concentrations. Some of the elements (e.g., towns) are connected directly to the plant, effectively providing a steady flow of wastewater. Some elements have a retainer tank between the wastewater source and the plant (e.g., meteorological wastewater). Finally, some elements present both options, they can discharge wastewater directly to the plant or store it on a retention tank (e.g., industries). The different elements are connected using pipes that form a sewage network. Some points in the network (e.g., where industrial wastewater is mixed with household wastewater) might be observed by sewage inspectors. Finally, a competent authority oversees the whole system taking decisions to ensure both the ecological quality of the water and the economic sustainability of the society.

Our agent-based model is built on top of the ALIVE (Aldewereld et al. 2010) framework. This Chapter focuses on the specification of ALIVE's organisational model, putting special emphasis on the norms and how they evolve due to organisational, technological, social and contextual changes.

The rest of this chapter is organized as follows. Section 2 describes the process followed, and the case study. It is an urban wastewater system inspired on the actual Besós river basin which is fully described in Section 2.1. In Section 3 we introduce the Tragedy of the Commons. Section 4 explores the objectives, roles and social structure of the system with the communication links. In Section 5 we introduce the basis and elements for the decision making from a wastewater management perspective. Later, Section 6 introduces the norms governing the system and provides examples of how they can evolve dynamically. Finally, Section 7, presents a discussion and the main conclusions of this chapter.

\section{Methodology}

In the ALIVE organizational model roles are the central concept. Roles identify the activities necessary to achieve organizational objectives and enable abstraction from the specific actors that perform them (Dignum and Dignum 2009). Based on these conceptualizations, the modelling process follows an iterative application of the following steps:

- identify the stakeholders in the system

- formally define the roles, identifying their goals and their dependencies

- model the interaction scenes between roles. Scenes are used to manage role dependencies via interaction protocols

- organize the scenes into a coherent interaction structure 
- identify the way agents will enact roles at run-time.

All this process is supported by the OperettA Tool (Aldewereld and Dignum 2010), this is one of the results of the ALIVE FP7 funded project (Vázquez-Salceda et al. 2010).

\subsection{Schema of wastewater flows in the case study}

The case study is a River Basin composed by elements generating both wastewater (a set of households $\left[\kappa_{1}, \ldots \kappa_{k}\right] \in \mathrm{K}$ and a set of industries $\left[I_{1}, \ldots I_{i}\right] \in \mathrm{I}$ ) and polluted water (meteorological events that generate runoff). For simplicity we will consider both wastewater and polluted water to be wastewater. There are also elements storing wastewater (a set of retention tanks $\left[T_{1}, \ldots T_{l}\right] \in \mathrm{T}$ ), treating wastewater (a set of Urban Wastewater Treatment Plants $\left[W_{1}, \ldots W_{j}\right] \in \mathrm{W}$ ), and receiving waters (e.g. a River).

Also, there is a graph $s_{i} \in \mathrm{S}$ that represents the sewerage infrastructure in a urban sector or city. It encompasses components such as receiving drains, manholes, pumping stations, storm overflows, and screening chambers of the combined sewer or sanitary sewer. $S_{i}$ ends at the entry to a $W_{j}$. In turn every $W_{j}$ is connected with the receiving waters. In our model as in many European countries all elements in $\mathrm{K}$ and $\mathrm{I}$ are obliged to connect their sanitation and/or wastewater discharge to $s_{i}$ where possible.

The wastewater is characterized by the flow (or volume) and the pollutant concentrations of: Total Suspended Solids (TSS ), Biochemical Oxygen Demand ( $B O D$ ), Chemical Oxygen Demand ( $C O D)$, Total Nitrogen $(T N)$ and Total Phosphorus (TP), which are defined as the set of pollutants $x_{r}$ with ( $T S S, D B O, C O D, T N, T P)=\left(x_{1}, x_{2}, x_{3}, x_{4}, x_{5}\right)$ (Verdaguer et al. 2012). All concentrations related to these pollutants are indicated with a supra-index $r$, with $r=1, \ldots, 5$. For subsequent paragraphs, this specification is not repeated in the text in order to avoid many repetitions.

The household generates a wastewater mass $M_{D} \in \mathrm{M}$ with a particular volume volume $\left(M_{D}\right)=V_{D}$, and a concentration for each pollutant $O_{j} \in \mathrm{O}$ concentration $\left(M_{D}, O_{j}\right)=C_{D}^{j}$, which is discharged in a plant $W_{k} \in \mathrm{W}$. Analogously, the runoffs retention tank has a wastewater mass stored $M_{M} \in \mathrm{M}$ with volume volume $\left(M_{M}\right)=L_{M}$ and pollutant concentration $\operatorname{concentration}\left(M_{M}, O_{j}\right)=C_{M}^{j}$. The tanks has a volumetric discharge to $W_{k}$ and feasible volumetric bypass to receiving waters when the retention tank has an 
overflow. It means the tank can bypass a water mass $M_{D M} \in \mathrm{M}$ with a volume $\operatorname{volume}\left(M_{D M}\right)=V_{D M}$. These two discharge possibilities allow adapting the sewer performance of separative or combined run-off collection. Each industrial activity has its own retention tank, with a water mass $M_{i} \in \mathrm{M}$ with volume $\operatorname{volume}\left(M_{i}\right)=L_{i}$ and pollutant concentration concentration $\left(M_{i}, O_{j}\right)=C_{i}^{j}$. Its volumetric discharge to the treatment is volume $\left(M_{i}\right)=V_{i}$. The plant $W_{k}$ is capable of accepting a water mass as influent $M_{T} \in \mathrm{M}$ with volume $\operatorname{volume}\left(M_{T}\right)=V_{T}$ and pollutant concentration concentration $\left(M_{T}, O_{j}\right)=C_{T}^{j}$. It provides a water mass as effluent $M_{e} \in \mathrm{M}$ with volume $\operatorname{volume}\left(M_{e}\right)=V_{e}$ and pollutant concentration concentration $\left(M_{e}, O_{j}\right)=C_{e}^{j}$ to receiving waters.

Additionally, the treatment has the possibility to bypass wastewater. The bypass consists in a water mass $M_{b} \in \mathrm{M}$ with volume volume $\left(M_{b}\right)=V_{b}$ and pollutant concentration concentration $\left(M_{b}, O_{j}\right)=C_{b}^{j}$. The upstream provides a water mass $M_{U} \in \mathrm{M}$ with volume volume $\left(M_{U}=V_{U}\right)$ and pollutant concentration concentration $\left(M_{U}, O_{j}\right)=C_{U}^{j}$ to receiving waters. The receiving waters correspond to a section of river basin, which has a water mass $M_{R W} \in \mathrm{M}$ with volume $\operatorname{volume}\left(M_{R W}\right)=V_{R W}$ and pollutant concentration concentration $\left(M_{R W}, O_{j}\right)=C_{R W}^{j}$

\section{Tragedy of the Commons}

The Tragedy of the Commons was described by Hardin (Hardin 1968), inspired by the lectures of Lloyd (Lloyd 1833) about population growth. The Tragedy is a situation where a set of appropriators consume a common good - or common-pool resource (CPR). These appropriators have incentives towards an over development or excessive exploitation of the common good, thus leading to depleting it. The basis of these incentives lays on the fact that increasing their consumption capacity brings them a direct benefit, while the cost of that increment is divided among all appropriators, thus the option of augmenting consumption capacity always beneficial. Assuming all agents are rational, everybody will. Hardin noticed that this problem cannot be solved by means of technical solutions, since it requires a change in the values and morality of people. That is why Hardin coined this as a Tragedy, not as in its common meaning of drama work, but as how the own rationality of agents lead to an inexorable fatal destiny. However, this is not necessarily what occur in this kind of scenarios.

Ostrom (Ostrom et al. 1994) presents a list of cases were the tragedy is avoided and highlights the case of how a South California basin area was heading to an 
overexploited scenario and how an institutional arrangement - by means of an equity court system and establishing special districts - to use basins allowed to not only prevent the tragedy, but ensured water availability even in significant drought periods. Hardin, then reviewed his position and clarified that the Tragedy occurs mainly in unmanaged commons (Hardin 2007). Ostrom also showed criticism towards solutions to manage CPR by means of central government institutions, that are far away from the local people who has to deal with the common good. According to her, the results are always suboptimal and temporary, since they are usually based on coercive measures and, if they are not accompanied of monitoring and sanction capabilities, instead of avoiding the tragedy, it is even fostered. Ostrom prefers self-organised institutions who devise its own rules, since it seems to be a common factor on long-live surviving CPR (Ostrom 1990) (Ostrom 1999).

Ostrom defines a CPR facility as an element which provides the conditions to sustain a stock of resource units. This stock produces a flow of resource units over time that can be appropriated (and diminished) as it is consumed (Ostrom et al. 1994). For instance, a fishing grounds and tons of fish, a windmill field and electricity. If the common good is renewable, it is possible to define a regeneration rate. While the amount of appropriated units does not exceed this regeneration rate, the CPR will be sustainable. If the common good is an exhaustible resource - no regeneration rate - or the appropriation exceeds the regeneration rate, the common goods will be eventually depleted.

With this representation of CPR, Ostrom also proposes to distinguish the problems of a CPR situation into two types (Ostrom et al. 1994): appropriation and provision problems. Each of them also poses additional sub-problems.

Appropriation problems refer to the development of rules to manage common good consumption: excluding potential beneficiaries and resource allocation from the resource flow produced by the CPR stocks - the main concern of this kind of problems is the flow of common good. In this kind of problems it is assumed that the relation between the yield provided by the CPR and the required inputs to obtain that yield, is given (see Fig. 1). 
TIME-DEPENDENT PROVISION PROBLEMS

YIELD IN TIME $t$ DEPENDS ON STRATEGIES IN TIME $t-1, t-2, \ldots$
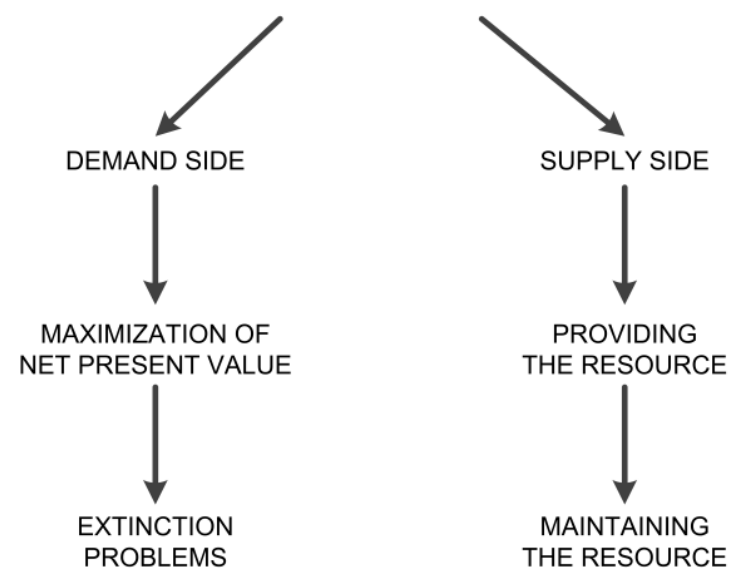

Fig. 1. A framework for provision problems (Ostrom et al. 1994)

\section{APPROPRIATION PROBLEMS}

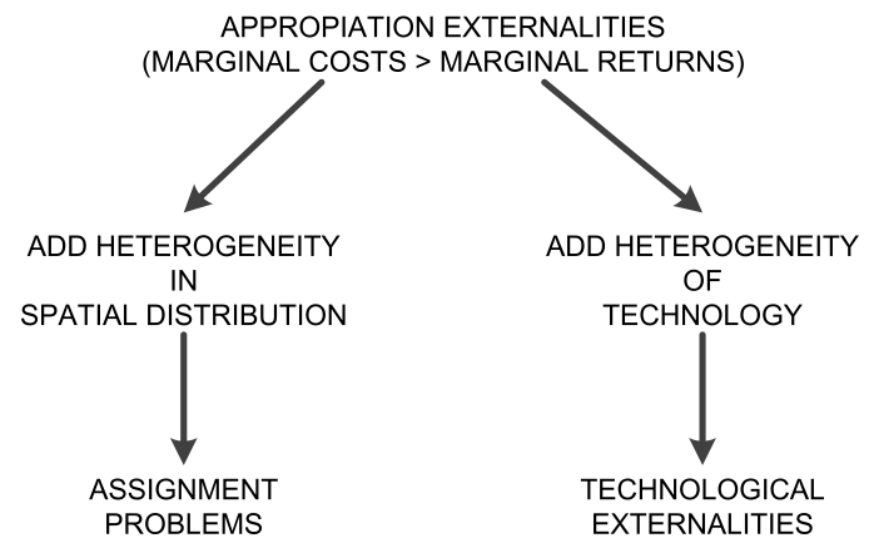

Fig. 2. A framework for appropriation problems (Ostrom et al. 1994)

Provision problems refer to the development of rules to manage contributions to: creating a resource, maintaining or improving its production capabilities and/or avoiding its destruction - the main concern of provision problems is the CPR stocks (see Fig. 2). 


\subsection{Designing institutions for CPR}

Institutions, understood as a set of agreed conventions on a community of agents, is relevant in the way common goods are managed. The rules that agents create or are imposed by external agencies, drive how appropriators interact with the common good which is a key factor on ensuring its sustainability.

Humans relate and interact with other people according to conventions or rules that have emerged from communities and society. All human societies devise constraints to structure and regulate the relationship between its members (Vázquez-Salceda 2003). Institutions are distinguishable by the set of constraints that govern these relations.

North studied the effect of institutions (which he considers a set of constraints) on the behaviour of human organizations. He points out that these institutional constraints ease the interaction among humans, shaping choices and making outcomes predictable (North 1990). The devising of these constraints allows for a growth on the complexity of the organizations while keeping interaction costs reduced, and similarly, allows the participants of the institution to act, and expect other participant to act according to a list of rights, duties, and protocols of interaction.

For this reason, the creation of Institutions provides trust among parties even when they do not have much information about each other. In environments with incomplete information, cooperative interactions can perform ineffectively unless there are institutions which provide sufficient information for all the individuals to create trust and to control deviations.

Institutions can be classified according to how they are created and maintained, or on the formality of its rules. On the former case, Institutions can be created from scratch and remain static or be continuously evolving. On the latter, institutions can be informal, that is, defined by informal constraints such as social conventions and codes of behaviour, or formal, defined by formal rules. Those can be political and legal rules, economic laws, or contracts.

In formal institutions the purpose of formal rules is to promote certain kinds of exchange while raising the cost of undesired kinds of exchange. Ostrom (Ostrom 1999) classifies formal rules in 7 types:

1. boundary rules affect the characteristics of the participants,

2. position rules differentially affect the capabilities and responsibilities of those in positions,

3. authority rules affect the actions that participants in positions may, must or must not do,

4. scope rules affect the outcomes that are allowed, mandated or forbidden,

5. aggregation rules affect how individual actions are transformed into final outcomes,

6. information rules affect the kind of information present or absent in a situation,

7. pay-off rules affect assigned costs and benefits to actions and outcomes. 
As norms are the elements that characterize institutions, they do not only serve as norms to be followed, but also serve as indication for people to recognize an organization as being an instance of a particular kind of institution, and then use this knowledge to predict other norms that could be applicable.

\section{Wastewater Systems' Organizational Model}

Agent based systems (Wooldridge and Jennings 1995) are an alternative for designing and implementing open and dynamic systems. As defined by Wooldridge \& Jennings: An agent is an encapsulated computer system that is situated in some environment and that is capable of flexible, autonomous action in that environment in order to meet its design objectives. Agents are capable of social behaviour, they can communicate, compete and cooperate among them.

The main idea behind a society is to allow its members to coexist in a shared environment and pursue their respective goals in cooperation or competition with others. Therefore, artificial social systems (Moses and Tennenholtz 1995) define an abstract social level over computational systems. The social level models the MAS as an organization of entities, defining structured patterns of behaviour that facilitate and enhance the coordination of agent activities (Vázquez-Salceda 2003), effectively providing an Organizational Model agent's can understand and use. We will use a Institution as the ones described in Section 3 to model agent's interaction in the Besòs River Basin.

This section briefly introduces the Organizational Model of our scenario, including the Social Structure (with roles and their relationships) the Interaction Structure (with the Landmarks, patterns of interaction by which agents coordinate their behaviour) and the Social model (mapping abstract roles to particular agents). For a in-depth discussion see (Gómez-Sebastià 2016).

\subsection{Social Structure}

ALIVE Social Structure allows the description of the roles and their relationships, connecting them with both the individual goals and the societal aims. In our proposal the global aim of the wastewater systems' organizational structure is to achieve an effluent with characteristics adequate to the quality requirements of receiving waters. The roles model the distribution of responsibilities among stakeholders and their dependencies. Fig. 3 shows the set of roles and their associated objectives and sub-objectives. For the sake of brevity we will not explain all of them but the most relevant. Role dependencies come in three wastewater, hierarchically dependencies (where the parent role has some form of authority over the child role, and therefore when the parent role requests the child role to perform a task, the child role is expected to abide), which are indicated with $H$ in Fig. 3, 
network dependencies (where roles coordinate themselves as peers by mutual interest and support each other to fulfil a common goal) and market dependencies (where there is a set of producer roles offering information and/or services to consumer roles for a given price), which are indicated with a $M$ in Fig. 3.

Also in Fig. 3 External Ex and Internal $I n$ roles are shown. Internal roles are controlled by the organization. Typically, if they are software components it means the organization has access to the software source code and is able to control and verify it. External roles are those participating in the organization, but not controlled by it. Following the same example, in the case of software components it means they have not been necessarily developed by the organization, and therefore there it might be no way to access the component's code and formally verify its behaviour.

The following list describes the most relevant roles in the system. Roles are depicted in bold while their objectives are in italics. Dependencies with other roles are also presented and, when relevant, the dependency relation is also introduced.

- IndustrialOperator: This role is aimed on industrial processes that generate economic revenue and, therefore, allow fulfilling the objective of making profit (MakeProfit). These industrial processes produce polluted water masses as a trade-off of their activity (Produce). Dealing with this wastewater requires the collaboration of a IndustrialWWRetainer which stores the wastewater to be discharged into the sewer system later on (StoreIndustrialWW). 


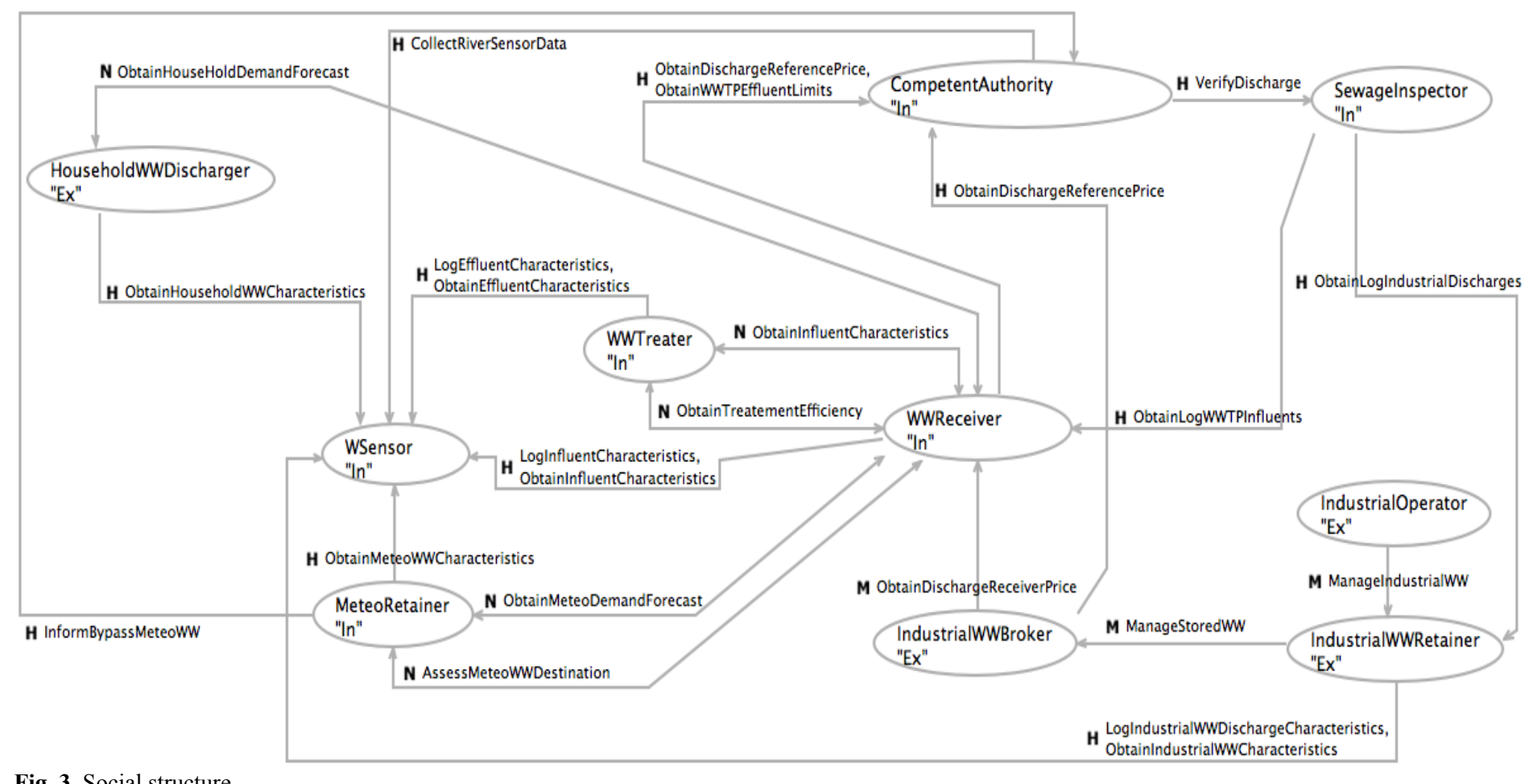

Fig. 3. Social structure 
- IndustrialWWRetainer: Stores wastewater produced by an IndustrialOperator (StoreIndustrialWW) and takes care of it (ManageStored $W W$ ) until it is possible to discharge it to the sewer system (DischargeIndustrialWW). To perform such discharge it is required the support of a IndustrialWWBroker to negotiate the discharge price and assess the feasibility of discharging some or all the wastewater, or keep storing it. Besides this, it keeps up a registry of all its industrial wastewater discharges performed (LogIndustrialWWDischargeCharacteristics), which requires the support of a WSensor to analyse the characteristics of discharged wastewater. This information can be used by a SewageInspector to verify I are properly managing discharges (VerifyDischarge).

- IndustrialWWBroker: Negotiates industrial wastewater discharges with a WWReceiver to assess how much wastewater is feasible to be discharged (AssessAmountOfIndustrialWWDischarge). From the IndustrialWWBroker perspective this assessment requires knowing its reserved cost (ObtainDischargeReservedCost) (i.e., how much $I_{i}$ is willing to pay according to the discharge price given by the WWReceiver). IndustrialWWBroker is consuming treatment capacity to discharge wastewater and comply with the policies and norms that regulate wastewater discharges and ensure water quality. WWReceiver is offering such service thus the relation is a consumer-provider one thus the dependency between both roles in the role dependency diagram is a market dependency.

- WWReceiver: Takes care of negotiating the reception of wastewater masses (NegotiateDischarge). This includes providing discharge prices for I (CalculateIndustrialWWDischargePrices) and the treatment capacity available for industrial wastewater (CalculateIndustrialWWAvailability). To calculate discharge prices the WWReceiver uses discharge reference prices provided by the CompetentAuthority, the current treatment efficiency in $W_{j}$ (ObtainTreatmentEfficiency) as well as the characteristics of the industrial wastewater that $I_{i}$ wants to discharge, which are provided by the IndustrialWWRetainer as part of the wastewater discharge negotiation process. Concerning treatment capacity availability, it depends on three main elements: Wastewater being received from households (ObtainHouseholdDemandForecast), meteorological retainers status (ObtainMeteoDemandForecast) and current $W_{j}$ effluent limits imposed by the

CompetentAuthority. Once influent is received, the WWReceiver determines its destination, either to be sent for treatment or bypass it directly to the river. This decision depends on (EvaluateInfluentDestination) wastewater characteristics (ObtainInfluentCharacteristics), $W_{j}$ current treatment efficiency (ObtainTreatmentEfficiency) and available capacity (CalculateAvailableCapacity). Finally, it also keeps a record of the influent characteristics received (LogInfluentCharacteristics) for the SewageInspector 
(as part of the VerifyDischarge task) and WWTreater (in order to calculate treatment efficiency).

- WWTreater: Processes the wastewater to reduce its pollutants concentration (WWTreatment). Once the treatments ends, treated water is discharged as an effluent to the river. This effluent is analyzed (ObtainEffluentCharacteristics) and information is logged so the CompetentAuthority can audit it (VerifyWWTPEffluent). Given the effluent and influent characteristics (ObtainInfluentCharacteristics / ObtainEffluentCharacteristics), WWTreater can calculate treatment efficiency (CalculateTreatmentEfficiency). This calculation is used to keep $W$ as efficient as possible (AchieveAdequatePerformance). It is also used to support WWReciever during discharge price negotiation described before.

\subsection{Interaction Structure}

ALIVE Interaction Structure allows the description of abstract patterns of interaction which are the way the roles coordinate their behaviour, managing their dependencies while they pursue their individual and collective objectives. The interaction structure defines interaction patterns known as scenes (Dignum and Dignum 2009) that allow actors to coordinate. The structure defines a set of scenes and transitions among them. On every scene one or more role dependencies (identified in the previous phase) are managed.

The structure's entry point is represented by a circle (init label), while the exit points are represented by triangles ( end label). Scenes are represented by rectangles and connected by lines (scene transition arcs) that allow the system to navigate from scene to scene. Inside every scene, the landmark patterns describe the protocol that must be used to achieve the scene result (Dignum and Dignum 2009). This diagram will focus on scene transition, allowing the following diagrams to focus on the different particular scenes by showing the landmark patterns inside them. The entry point leads to the different wastewater generation scenes (Household, MeteoWWGenerate and IndustryWWGenerate) and the scenes related to water quality protection by the competent authority (EnforceWaterQualityPolicies, ComputeWWTPEffluentLimits, ComputeIndustryPermissionLimits and ComputeDischargeReferencePrices). HouseholdWWGenerate leads directly to wastewater treatment scenes. MeteoWWGenerateScene contain particular scenes for discharging the water to $\mathrm{W}$ (MeteoWWDischarge) or bypassing it (MeteoWWBypass). Industrial production is divided in two parts: first wastewater is generated and stored IndustryWWGenerate and, later on, a negotiation to discharge wastewater in tank is done (IndustryManageWWTank) where a price to discharge is formed (WWTPAssessDischargePrice); depending on the negotiation result 
(IndustryAssessWWDischarge) wastewater is discharged (IndustryWWDischarge) or it is kept.

Some discharges will have a discharge verification performed asynchronously by the competent authority (VerifyDischarge). Wastewater treatment scenes include receiving influent (WWTPReceiveInfluent) and either treating (WWTPTreatInfluent) or bypassing it (WWTPBypassfluent)).

Fig. 4 depicts the internal landmark patterns of the scenes WWTPTreatInfluent and WWTPBypassfluent.
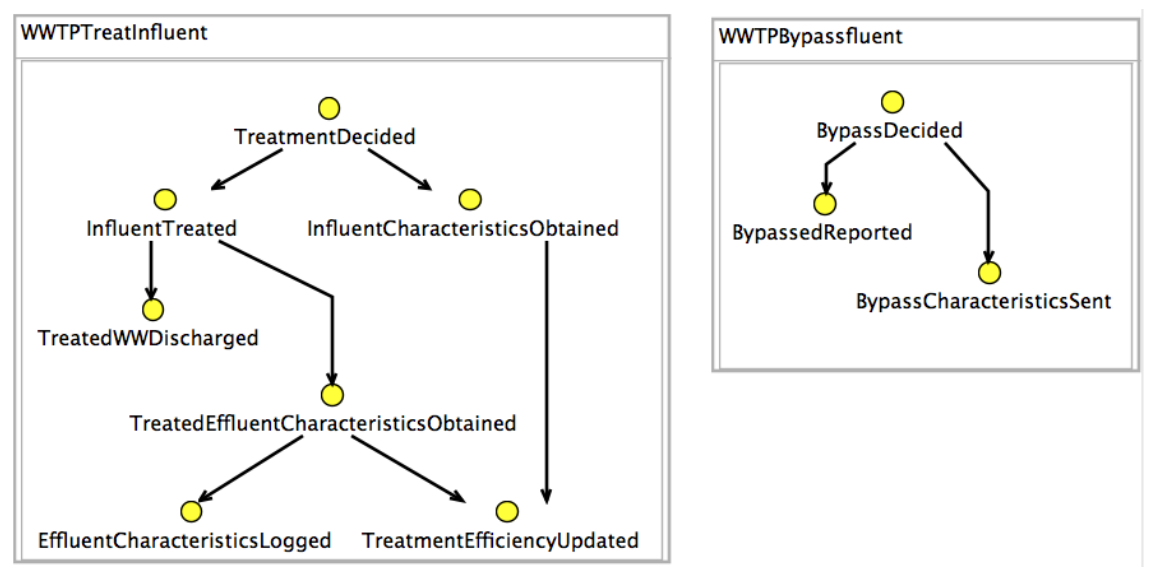

Fig. 4. Landmark patterns for WWTP influent treatment

\subsection{Social Model}

Up to this point the overall system has been defined in terms of roles, their aims and dependencies, without taking into account who will actually enact those roles at run-time. The Social Model defines the way concrete agents enact the organization roles, guiding their behaviour and achieving coordinated action by following the organizational patterns established for the roles they enact. An agent can enact one or several roles, depending on their objectives, and one role can be enacted by more than one agent. In the case study, the objectives of UWS infrastructures act as the main driving force for allocating roles into agents.

Fig. 5 shows the model of agents. The central axis is composed by the types of agents with a Household, Meteorological, Industrial, WWTP and River Council agents (square-shaped). The surrounding nodes represent the roles (round-shaped). The arrows connect each role with the agents type that performs it. 


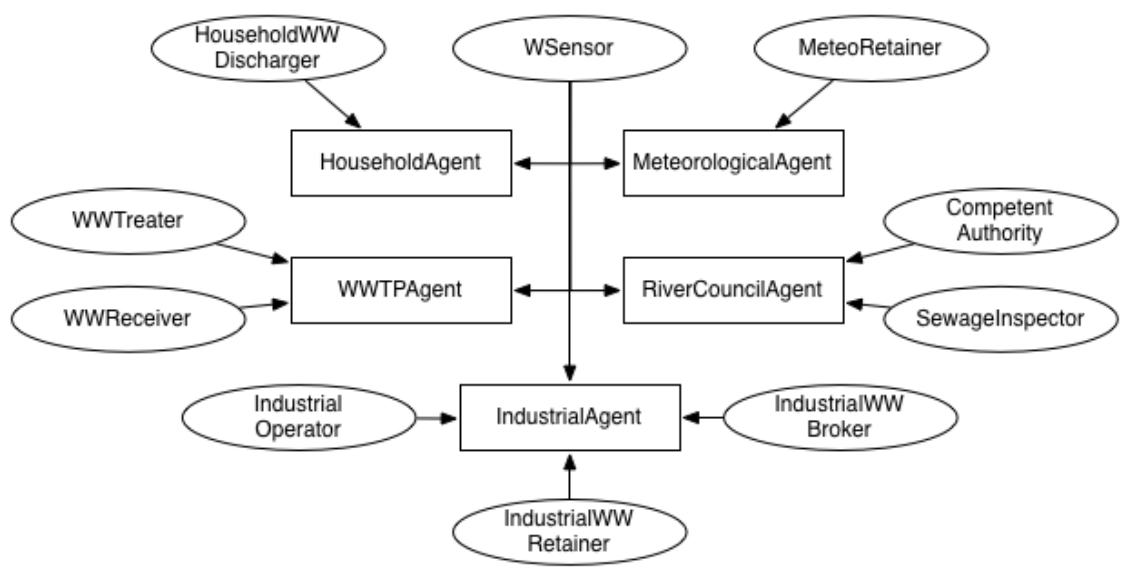

Fig. 5. The model of agents describes the roles that each kind of agent can enact during its lifetime.

\section{Agents: Behavior and decision-making}

The most important roles in our system, from an integrated management perspective, are two. First, the Competent Authority is responsible for all matters relating to the collection, treatment and disposal of wastewater. Second the $W_{k}$ which is responsible for treating wastewater in that sector or city. In this section we focus on the latter to describe the decisions it has to make to carry out the negotiation with industries that would like to discharge the wastewater resulting from their activities. The discharge of a wastewater mass requires an agreement between a particular and a particular that can accept it for treatment. This process requires knowing whether a $W_{j}$ is capable to properly handle the proposed wastewater discharge.

We define the water characteristics of a water mass $M_{k}$ as the pair $(V, C)$, where: $V$ is the volume of water mass given in cubic meters $m^{3}$ and, $C$ is the pollutant concentration in the water mass. We also define $C=\left(C^{1}, \ldots, C^{r}\right)$ as the set of pollutants concentrations ${ }^{1}$ where each $C^{i}$ corresponds to a specific pollutant concentration $(1 \leq i \leq r)$. In our system, as already explained in Section 2.1, we consider five different pollutants: (TSS, BOD,COD,TN,TP). Thus $C=\left(C^{1}, C^{2}, C^{3}, C^{4}, C^{5}\right)=(T S S, B O D, C O D, T N, T P)$ (Verdaguer et al. 2012).

${ }^{1}$ Pollutant concentration is given in $\left(\frac{\mathrm{kg}}{\mathrm{m}^{3}}\right)$. 
Given these premises, the negotiation process between $I_{i}$ and a $W_{j}$ can be described as follows: The $W_{j}$ checks if it can manage the wastewater that the industry wants to discharge (a $W_{i}$ characterized as $\left(V_{i}, C_{i}\right)$ ). This means ensuring there is enough physical space to receive it (volume availability) and that the plant can effectively treat the pollutants contained in the wastewater mass (pollutant concentration admissibility); if wastewater contains a high pollutant concentration it can harm the treatment process since it depends on bacteria colonies that may perish.

Volume availability ( $\left.V_{\text {available }}\right)$ depends on the design volume of the $W_{j}($ $\left.V_{\text {capacity }}\right)$, the amount of domestic wastewater sent by households $\left(V_{d}\right)$ and meteorological phenomena $\left(V_{m}\right)($ e.g., rain), whose treatment is mandatory. Finally, previously agreed industrial discharges $\left(V_{\text {scheduled }}\right)$ have to be taken into account to know what volume capacity remains available for new industrial discharges:

$$
V_{\text {available }}=V_{\text {capacity }}-V_{d}-V_{m}-V_{\text {scheduled }}
$$

Therefore, if $V_{i} \leq V_{\text {available }}$ then there is enough space in the $W_{j}$ to accept the wastewater mass.

To verify pollutant concentration admissibility the process is similar although a $W_{j}$ can admit a higher concentration than the one it can effectively manage; however, this will imply a significant higher cost for the industry. Pollutant concentration admissibility depends on how much pollutant concentration $W_{j}$ can manage as a parameter design of the plant $\left(C_{\text {admissible }}^{r}\right)$. Thus, if $C_{\text {admissible }}^{r} \geq C_{i}^{r}$ the wastewater mass will be accepted without extra cost. Otherwise, the pollutant overload will carry an extra cost to the price that industry has to pay. This price is calculated by $W_{j}$ as follows:

$$
P\left(W W_{i}, W_{j}\right)=P\left(\left(V_{i}, C_{i}\right), w w t p_{j}\right)=V C\left(V_{i}, W_{j}\right)+P C\left(W W_{i}\right)
$$

Eq. 2 is divided into two parts: a volumetric cost and a pollutant cost. The first represents the cost of accepting a certain volume of wastewater generated by $I_{i}$ according to the current state of the $W_{j}$ as well as the taxes defined by the competent authority. The second computes the price of processing the wastewater discharged by industry $I_{i}$ according to its pollutant concentration. 


\section{Norms}

Scenarios like the one introduced in this Chapter present several actors (e.g., Industrial Operator, Industrial WWRetainer, WWTreater etc. ) with a variety of goals that sometimes are conflicting between them. For instance, the Industrial Operator aims at making profit which in turn will generate wastewater, effectively polluting the environment. The WWTreater aims at cleaning wastewater for protecting the environment at the lowest possible price. Therefore, from the individual point of view of an Industrial Operator, the more industrial activity the better, even if it results in more polluted water. However, from the individual point of view of a WWTreater the less industrial activity the better, as the water will be less polluted and therefore will be easier and cheaper to clean. Bringing this self-interest to the extreme, the ideal situation for Industrial Operator is a scenario with no environmental protection, where the river can be polluted without constraints. The ideal situation for WWTreater is a scenario where there are no polluting elements (no industries, no households) and therefore the river is never polluted. However, the ideal situation from the holistic point of view of the society (as a group of interconnected individuals) is to find a balance to protect the environment while promoting industrial activity.

Furthermore, the roles in the scenario depend on each other for achieving their goals, and therefore they interact in multiple ways. The combination of these two factors results in a society of interacting agents with heterogeneous goals. In order to tame the complexity of these interactions, and to align the overall system with a common high level goal (e.g., protecting the environment without compromising industrial activity) norm-aware electronic distributed systems can be used.

Electronic specifications of norms are one of the mechanisms being applied to define and enforce acceptable behaviour of electronic distributed systems which should comply with some (typically human) regulations. One of the options for providing norm-aware MAS are Electronic Institutions (EI) (Vázquez-Salceda 2003). They are models of human institutions with a norm specification provided in a machine-readable formalism. The main idea behind $E I$ is capturing the essence of an institution (mainly norms and protocols) in a machine processable form.

Some functionalities in the system depend on the Competent Authority role detecting exceptional situations and applying the appropriate restrictions. For instance, if a meteorological overflow is notified WWPTs can exceed their treatment capacity if they take water from the influent. Therefore plants must limit their influent intake until the overflow is solved. It means water from the influent is not completely treated and to protect the environment industries can not discharge wastewater to the river until the overflow is solved. In order to tackle these restrictions we use ALIVE 's normative structure, grounded on regulative and constitutive norms. Such norms specify actor's obligations (WWTPS must limit influent intake until the overflow is solved), prohibitions (discharging water masses with high concentrations of mercury) and permissions (industries can not discharge wastewater to the river until the overflow is solved). In scenarios like the one 
presented here, MAS are applied to systems with an overall holistic goal and it is not desirable that an agent's autonomous and emergent behaviour diverges from the overall goal of the system. In order to limit this agent autonomy and ensure a certain coherence between the goals of the particular agents and the overall goals of the system, norms can be applied. Furthermore, norms make the behaviour more predictable, effectively reducing the complexity of the system. Taking this into account, the scenario presented in Section2.1 provides an exciting new line of research: modelling and implementing the set of norms that will make the system's objectives ( e.g., have an environmental sustainable system) stand on top of individuals' objectives (e.g., make profit in the case of the industries). Furthermore, the set of norms provided is not static, as norms will have to evolve through time just as individuals' behaviour changes to adapt to dynamic circumstances. Not only deciding how these norms will have to be adapted is an exciting challenge, but also designing mechanisms to support norm dynamics at run-time. Such mechanisms effectively support adding, removing or updating norms at run-time and while inferring the social state. On the one hand, we can not afford to miss the violation of a norm just because we are updating it. On the other hand, we have to infer a social state consistent with the changes performed in the norms (e.g., it makes no sense to punish an agent for violating a norm that has been removed).

This section provides examples of norms modelled used the framework introduced in (Gómez-Sebastià 2016) and examples on how social and technological changes can affect these norms. For each norm a formal model is provided, as well as the time line depicting the implications of the norm change. Examples for all operations supported by our framework are provided. Norms are inspired on European, national and local wastewater treatment directives.

Our model supports two possible operations (adding and removing norms, accounting norm update as a combination of the two basic operations) in two forms (retroactively and prospectively). The model also supports regulative norms (with obligations, prohibitions and permissions), constitutive norms and institutional powers (including both constitutive powers and normative powers).

\subsection{Obligation prospective add:}

The European council directive for Wastewater treatment (European Council 1991) in Article 4 and the Catalan plan for Wasterwater treatment inspired on this directive (Generalitat de Catalunya 2005) state:

Member States shall ensure that urban wastewater entering collecting systems shall before discharge be subject to secondary treatment or an equivalent treatment as follows:

- at the latest by 31 December 2000 for all discharges from agglomerations of more than 15000 p.e. (population equivalent) 
- at the latest by 31 December 2005 for all discharges from agglomerations of between 10000 and 15000 p.e.

It means that, by the date ' 01 January 2006' each $W W T P_{i} \in \mathrm{W}$ with a p.e. of 10.000 or more have the obligation to perform a secondary treatment (or a treatment that counts-as secondary treatment, that is, an equivalent) before discharging water to the river. Failing to comply with the norm will result in the WTTP being sanctioned. Fig. 6 shows the formal specification of the regulative norm in our model.

\begin{tabular}{l} 
Norm $N_{1}:$ Let $W_{i} \in \mathcal{W}$ be a Wastewater Treatment Plant, $M_{j} \in \mathcal{M}$ a water mass and \\
$T_{k} \in \mathcal{T}$ a secondary treatment. Once $W_{i}$ receives a particular water mass $M_{j}$, the plant \\
has the obligation to treat the water mass with secondary treatment $T_{k}$ before discharging \\
the water mass. \\
Sanction $S_{1}:$ A generic sanction is applied to the Wastewater Treatment Plant if the norm \\
is not complied with. \\
\hline \begin{tabular}{|l|l|}
\hline Activation Condition $N_{1}$ & received $\left(W_{i}, M_{j}\right)$ \\
\hline Expiration Condition $N_{1}$ & discharged $\left(W_{i}, M_{j}\right)$ \\
\hline Maintenance Condition $N_{1}$ & True \\
\hline Deadline $N_{1}$ & performed $\left(T_{k}, W_{i}, M_{j}\right) \wedge$ counts_as $\left(T_{k}\right.$, SecondaryTreatment $)$ \\
\hline Activation Condition $S_{1}$ & isViolated $\left(N_{1}, W_{i}\right)$ \\
\hline Expiration Condition $S_{1}$ & GenericSanction $\left(W_{i}\right)$ \\
\hline Maintenance Condition $S_{1}$ & True \\
\hline Deadline $S_{1}$ & True \\
\hline
\end{tabular}
\end{tabular}

Fig. 6. Example of formal norm specification for obligation

Following the example, the regulative norm is introduced in the system via a Prospective Promulgation operation on the date ' 01 January 2006'. Therefore, if a $W_{i} \in \mathrm{W}$ with a p.e. of 10.000 or more violated the regulative norm (i.e. discharged water without treating it) before '01 January 2006', the act has no legal consequences. However, if the plant violates the norm after the promulgation date, it will be sanctioned for the act. In the example depicted in Fig. 7, $W_{i}$ discharges untreated water masses $M_{1}, M_{2}$ before norm promulgation without legal consequences. However, discharging untreated water $M_{3}$ after promulgation results in a sanction being applied.

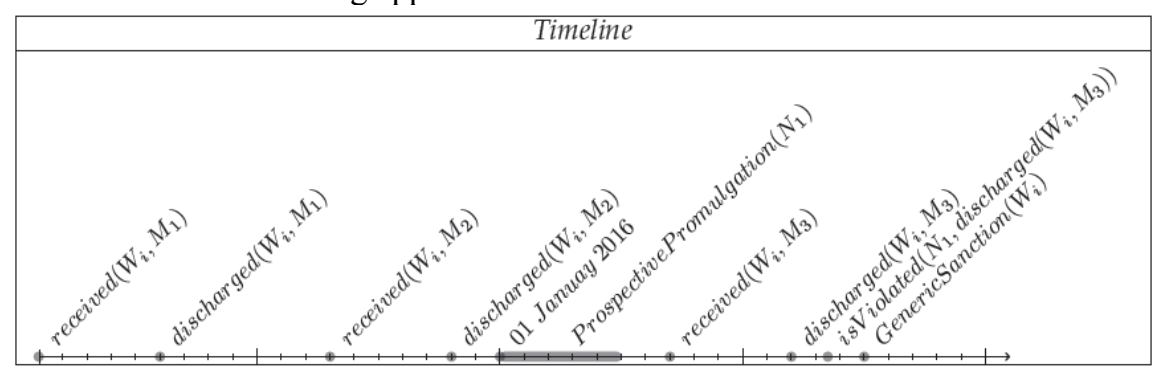

Fig. 7. Example of timeline for prospective add of a an obligation 


\subsection{Obligation prospective remove:}

The European council directive for Wastewater treatment (European Council 1991) states in Annex I point D.5:

Extreme values for the water quality in question shall not be taken into consideration when they are the result of unusual situations such as those due to heavy rain.

In our example it stands for the obligation to perform a secondary treatment not having effect in unusual situations, such as heavy rain. The obligation has already been introduced in Section 6.1 and formally modelled in Fig. 6. However, the model does not take into account the fact that the norm is not in place in case of unusual situations. One option is to include the exception on the model of the norm (i.e. preventing the norm from activating if water is received by the plant, but an unusual situation is in place). Formally, it would imply substituting the activating condition of Norm $N_{1}$, which is currently received $\left(W_{i}, M_{j}\right)$ for

received $\left(W_{i}, M_{j}\right) \wedge \neg$ unusualSituation () . However, this solution would result in more complex norm formalizations. Furthermore, if new exceptions to the norm are added, more conditions would be included in the activating condition, resulting in complex and hard to understand norms. A cleaner solution is to allow the competent authority (or any other actor with power to alter the norms that govern the system) to temporally remove the norm from the system when it is considered appropriate (in our example, while the unusual situation takes place). On the one hand, these norm could be used to keep norms simple and easy to understand. That is because we are leaving the decision of which norms should be active in every scenario to higher level (and more expressive) reasoning processes performed by the agents responsible of introducing and removing norms in the system. On the other hand, all the exceptions to the different norms do not have to be taken into consideration at norm design time. They can be introduced later when designing a process that decides which norms are active in the system at every point of time. That is, our approach allows supporting a normative system which is truly dynamic and adapts to changing (and sometimes even not foreseen) situations.

Following the example depicted in Fig. 8, the norm $N_{1}$ is removed from the system via an Abrogation operation. This allows to effectively implement a general exception to the norm while an unusual situation of heavy rain takes place. Therefore, if a particular Wastewater Treatment Plant $W_{i} \in \mathrm{W}$ violated the norm ( i.e. discharged water without treating it) in a situation of heavy rain, the act has no legal consequences. However, if the plant violates the norm outside the unusual situation, it will be sanctioned for the act. In this example, a Wastewater Treatment Plant $W_{i}$ discharges untreated water masses $M_{2}$ during heavy rain without legal consequences. However, discharging untreated water $M_{1}, M_{3}$ outside the unusual situation results in a sanction being applied. Please note that one of the sanctions 
(associated to the discharge of $M_{1}$ ) is applied during the unusual situation. This is because the action causing the norm violation occurred outside the unusual situation, and our framework is expressive enough to detect this particular fact.

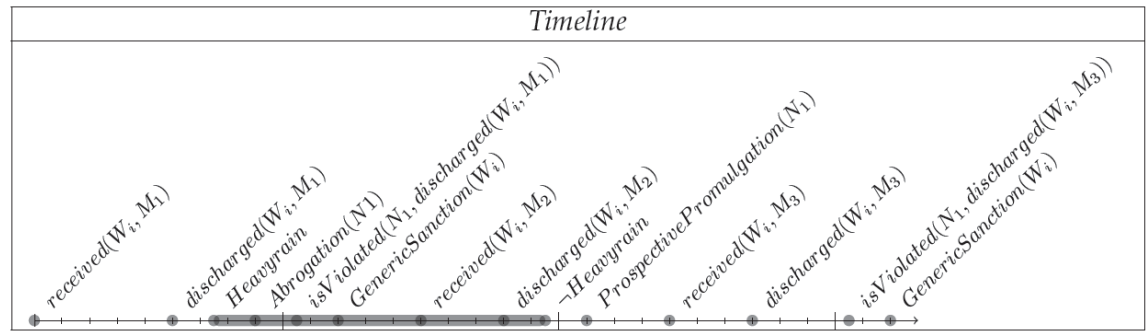

Fig. 8. Example of timeline for the prospective remove of an obligation

\section{Conclusions and Related work}

Once we have finished introducing our approach of a norm-aware multi-agent simulator, we proceed to put it in contrast with similar approaches, focusing on detecting confluence points where our work can be complemented by the different approaches in the state of the art. Then, we will outline conclusions.

The work in (Verdaguer et al. 2012) aims at coordinating coordinating industrial wastewater discharges between different actors, based on ant colony optimization. The system aims at finding the best combination of industrial discharges w.r.t. WWTP efficiency, that is, as much capacity as possible is used from the plant without overloading it. For doing it, ants are randomly placed on a graph-like search space, where nodes are industrial activities and edges possible discharges. The work neglects some issues such as the efficiency of a centralized decision system in real-world scenarios, or the need to have complete information about industry production plans in order to entail expected industrial wastewater discharges. Furthermore, the work presented in (Verdaguer et al. 2012) does not take into account agents not abiding to the expected patterns of behaviour, and lacks methods to deal with such issues.

The work in (Verdaguer et al. 2012) focuses on the internal reasoning process of a coordinator agent (which can be fulfilled by the WWTP agent in our scenario) whereas our approach focuses on the structure of the agent society and the interactions among agents from an organizational point of view, without detailing the internal reasoning processes of the agents. Therefore, we could state both approaches are complementary and can benefit from each other. The work in (Verdaguer et al. 2012) could benefit from the normative system we propose for enforcing acceptable patterns of behaviour (e.g., industries comply with the agreements they reach with WWTPs for wastewater treatment) as well as from our institutional model to facilitate coordination in complex scenarios where a particular agent can fulfil more than one role (e.g., industries with their own WWTP 
that can accept other industry wastewater as long as they are paid enough for treating it).

Market-based approaches are another alternative to coordinate the use of natural resources in heteronegous societies of actors. In (Garrido et al. 2013) $m$ Water is introduced. $m$ Water is a regulated virtual market simulation where autonomous agents trade rights for the use of water in a closed basin. The idea behind

$m$ Water is allowing policy makers to compare different market configurations using market performance indicators. Market configurations contain several parameters, including participant population (supporting different behavioural templates) and the set of regulations and protocols to be used during the negotiation process. Just like our approach, $m$ Water aims at narrowing the gap between water management simulations (based on equational descriptions) and social simulations. The motivation behind social simulations is to mimic the behaviour of autonomous rational individuals and groups of individuals (Smajgl et al. 2009). The main idea is modelling not only hydraulic factors (which can be perfectly modelled using equational systems) but also social factors, including norm typology and actor's behavioural templates.

$m$ Water and our proposal have several characteristics in common. Both are social simulations grounded on Electronic Institutions able to represent roles, coordination scenes, objectives and a normative system. However, when compared to our approach $m$ Water presents a more specific and in-depth proposal. $m$ Water focuses on negotiation for water use rights, whereas our proposal covers the whole river basin management scenario, therefore the negotiation process is not presented with such detail. Furthermore, while $m$ Water correctly emphasises the need to flexible and dynamic normative systems ( e.g., authors stress the need of 'organization schemes that are flexible and able to adapt to a changing environment with multiple situations') no method for supporting them is presented. Our proposal clearly remarks that this method is available, and we provided an exhaustive set of examples based on river basin management. On the one hand, we consider our proposal could benefit from the work done in $m$ Water for implementing more expressive and powerful auction mechanisms when negotiating for wastewater treatment resources. On the other hand, we consider $m$ Water could benefit from our proposal to widen the application scenario (limited not only to interactions involved on the negotiation for water use rights, but covering the whole set of interactions present in river basin management) and support dynamic normative systems, able to change the set of norms during the simulations, effectively adapting them to new situations and requirements. This would allow to simulate not only new sets of policies but also sets of policies evolving through time, allowing to evaluate not only the impact of the new set of policies in the system, but also the performance of the evolution ( e.g., measuring how long does it take for the new policies to be adopted and the performance of the system during the transition between different sets of policies). In general we consider evaluating the impact on the system of policy evolution, while the simulation keeps running and 
the different actors pursuing their objectives, opens new, more realistic and exciting lines of future work w.r.t. simulations for policy optimisation.

The work presented in (Ernst et al. 2007) presents a social simulator integrated with a DSS where a set of heterogeneous agents interacts. We like the idea of merging different agent behaviours and perceptions (including environmental, social and legal perceptions) via a 5 step reasoning process. However, we consider the approach could benefit from a clearly defined social model integrated in the reasoning process via socially-aware reasoning methods. Furthermore, it might be interesting to integrate protocols and regulations in the reasoning process (e.g., in the form of sanctions for agents consuming too much water in drought situations).

The work presented in (Gailliard et al. 2014) proposes a model for river basin governance that includes what is known as a 'boundary worker'. Boundary workers, such as river basin managers, are interfaces aimed at facilitating an evolution towards more sustainable practices in river-basin governance. The main aim of (Gailliard et al. 2014) is to analyze the impact of boundary workers on the behaviour of heterogeneous actors interacting on a common social-hydrosystem. This is achieved by creating an agent-based model including qualititative data (research questions, expected rules of behaviour, interactions between agents and some scenarios to simulate) which can be reviewed via a return field in order to calibrate the model as finely as possible. In contrast to (Gailliard et al. 2014) our approach supports governance either via a pre-defined set of rules (e.g., provided by a river basin manager) or via a consensual agreement between the different actors involved in the scenario. In any case, this set of rules will not be static, but will evolve through time as new regulations and behaviours emerge in the society of actors involved in river basin management.

To conclude, this chapter has presented a norm-aware agent-based model for integrated wastewater management systems. The chapter provides an example on how normative systems can be integrated in multi-agent systems where actors' objectives are heterogeneous and sometimes conflicting. The normative system allows to align agent's objectives with common organisational objectives. At the same time, it allows to detect undesirable patterns of behaviour in the agents, such as free raiders. Thanks to our proposal, misbehaving actors can be sanctioned, effectively enforcing good practices among the actors.

In this aspect, our proposal shows many features in common with several works in the state of the art. However, our proposal goes beyond, as it allows the set of norms governing the multi-agent system to evolve through time. We provide a wide range of examples, where regulative norms in the form of obligations, prohibitions and permissions are inserted, removed and updated. Furthermore, we also show examples of dynamic operations on constitutive norms and constitutive powers.

While most of the systems analysed show a less expressive normative language (they typically do not account for constitutive norms and constitutive powers) we provide a rich set of normative elements (Alvarez-Napagao 2016), supporting deontic elements (obligations, prohibitions and permissions), constitutive norms, constitutive powers and violation handling norms (i.e. sanctions). Furthermore, our 
normative elements contain a rich structure with activation, maintenance and deactivation conditions, as well as deadlines.

Finally, we support norm dynamics, which is not supported by the proposals analysed in the state of the art. We propose four operations to update the normative system accounting for norm promulgation and derogation both in prospective and retroactive forms. On the one hand, we combine norm operations with a rich set of normative elements providing a dynamic normative language that can be adapted to a numerous set of contexts and situations. This is specially important in wastewater management scenarios, where the set of norms will evolve adapting to situations which are typically out of control of managers and legislators (e.g., heavy rains, droughts, pollution of the environment). On the other hand we can adapt norms while our system is on-line, inferring a normative state consistent with the update. In scenarios like wastewater management we can not afford to stop observing the social reality, as free raiders and other misbehaving actors could take advantage of this situation.

In contrast, our proposal does not present complex reasoning processes and decision taking mechanisms for the agents involved in the system. We focus on the normative system, so we can effectively benefit from more expressive and complex agents the other proposals include. As a future research direction we plan to integrate a norm reasoning process into our simulator. This will allow the agents to decide which norms should be changed and how. Our hypothesis is agents can adapt norms to the scenario in order to regulate the ussage of natural resources. The objective is to avoid or delay the tragedy of the commons from ocurring.

As a summary, in this chapter we have seen a wide range of norms and norm operations, based in real world regulations and protocols, such as (European Council 1991). This chapter focuses on how the normative system can evolve. On the one hand adapting itself to new regulations and protocols caused by technological advances or social changes. On the other hand, adapting itself to unexpected situations which are typically out of control of managers and legislators, such as heavy rains. Furthermore the work presented in this chapter is being integrated into a social simulation system that models the Besòs river basin. In such simulation agents participate in a Tragedy of the Commons scenario where water quality represents the common good. Agents face the dilemma of breaking the rules to increase their utility functions or act legally according to current state of norms. Including normative dynamics allows us to adapt norms to environmental changes and see if those changes allow the common good to be preserved despite agents' behaviours and selfish interests that drives them to, under certain circumstances, act as free riders.

Acknowledgments Prof. Cortés is a member of the Sistema Nacional de Investigadores (México). 


\section{References}

Aldewereld, H. and Dignum, V., (2010). OperettA: Organization-oriented development environment. In Languages, Methodologies, and Development Tools for Multi-Agent Systems (pp. 1-18). Springer Berlin Heidelberg.

Aldewereld, H., Padget, J., Vasconcelos, W., Vázquez-Salceda, J., Sergeant, P. and Staikopoulos, A., (2010). Adaptable, organization-aware, service-oriented computing. In Intelligent Systems, IEEE, 25(4), pp.26-35.

Alvarez-Napagao, S. (2016) Bringing Social Reality to Multiagent and Service Architectures. Practical reductions for monitoring of Deontic-Logic and Constitutive Norms. PhD Dissertation Universitat Politècnica de Catalunya-BarcelonaTech.

Dignum, F., Dignum, V., Padget, J. and Vázquez-Salceda, J., (2009), December. Organizing web services to develop dynamic, flexible, distributed systems. In Proceedings of the 11th

International Conference on Information Integration and Web-based Applications \& Services (pp. 225-234). ACM.

Ernst, A., Schulz, C., Schwarz, N. and Janisch, S., (2007). Modelling of water use decisions in a large, spatially explicit, coupled simulation system. In Social simulation: Technologies, advances and new discoveries, pp.138-149.

European Council. (1991) Directive concerning urban waste water treatment (91/271/EEC), volume 30

Gailliard, N., Barreteau, O. and Richard-Ferroudji, A., (2014). Participatory Policy Making in Practice: Simulating Boundary Work in Water Governance. In Advances in Social Simulation (pp. 333-342). Springer Berlin Heidelberg.

Garrido, A., Giret, A., Botti, V. and Noriega, P., (2013). mWater, a case study for modeling virtual markets. In Agreement Technologies (pp. 565-582). Springer Netherlands.

Generalitat de Catalunya. Psaru (2005).

http://aca-web.gencat.cat/aca/appmanager/aca/aca?.nfpb=true

pageLabel=P1204654461208200526170 profileLocale=es Accessed: 2015-03-30.

Gómez-Sebastià, I. (2016) NoMoDEI A framework for Norm Monitoring on Dynamic Electronic Institutions. PhD Dissertation. Universitat Politècnica de Catalunya-BarcelonaTech. http://futur.upc.edu/17497419 Accessed 03 May 2016

Hamilton, H.R., (1969). Systems simulation for regional analysis: an application to River-Basin planning. Cambridge, Mass./London: Mass. Inst. Technol., 1969.

Hardin, G., (1968). The tragedy of the commons. In Science, 162(3859), pp.1243-1248.

Hardin, G., (2007). The tragedy of the unmanaged commons. In Evolutionary perspectives on environmental problems, pp.105-107.

Lloyd, W.F., (1833). Two Lectures on the Checks to Population: Delivered Before the University of Oxford, In Michaelmas Term 1832. JH Parker. 
Moses, Y. and Tennenholtz, M., (1995). Artificial social systems. In Computers and Artificial Intelligence, 14, pp.533-562.

North, D.C., (1990). Institutions, institutional change and economic performance. Cambridge university press.

Ostrom, E., Gardner, R. and Walker, J., (1994). Rules, games, and common-pool resources. University of Michigan Press.

Ostrom, E., (1990). Governing the commons: The evolution of institutions for collective action. In Political Economy of Institutions and Decisions. Cambridge U. Press, Nov. 1990.

Ostrom, E., (1999). Coping with tragedies of the commons. In Annual review of political science, 2(1), pp.493-535.

Ostrom, E., (2000). Collective Action and the Evolution of Social Norms.In Journal of Economic Perspectives, 14(3), pp.137-158.

Poch, M., Comas, J., Rodriguez-Roda, I., Sanchez-Marre, M. and Cortés, U., (2004). Designing and building real environmental decision support systems.In Environmental Modelling \& Software, 19(9), pp.857-873.

Smajgl, A., Heckbert, S., Ward, J. and Straton, A., (2009). Simulating impacts of water trading in an institutional perspective.In Environmental Modelling \& Software, 24(2), pp.191-201.

Vázquez-Salceda, J., Vasconcelos, W.W., Padget, J., Dignum, F., Clarke, S. and Roig, M., (2010). ALIVE: an agent-based framework for dynamic and robust service-oriented applications. In Proceedings of the 9th International Conference on Autonomous Agents and Multiagent Systems: volume 1-Volume 1 (pp. 1637-1638). International Foundation for Autonomous Agents and Multiagent Systems.

Vázquez-Salceda, J., (2003). The role of norms and electronic institutions in multi-agent systems applied to complex domains. The HARMONIA framework. In Ai Communications, 16(3), pp.209-212.

Verdaguer, M., Clara, N. and Poch, M., (2012). Ant colony optimization-based method for managing industrial influents in wastewater systems. In AIChE Journal, 58(10), pp.3070-3079.

Wooldridge, M. and Jennings, N.R., (1995). Intelligent agents: Theory and practice. In Knowledge engineering review, 10(2), pp.115-152. 